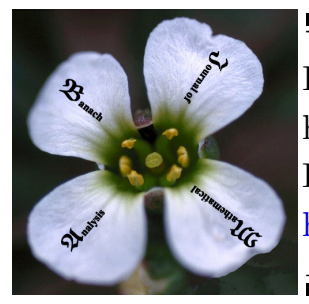

Banach J. Math. Anal. 9 (2015), no. 2, 253-275

http://doi.org/10.15352/bjma/09-2-17

ISSN: $1735-8787$ (electronic)

http://projecteuclid.org/bjma

\title{
SOME TYPES OF CONVERGENCE RELATED TO THE RECONSTRUCTION PROPERTY IN BANACH SPACES
}

\author{
G. KHATTAR AND L. K. VASHISHT* \\ This paper is dedicated to Professor S. K. Kaushik
}

Communicated by D. R. Larson

\begin{abstract}
Casazza and Christensen [Canad. Math. Bull., 51 (2008), 348358] introduced and studied the reconstruction property in Banach spaces. In this paper, we discuss different types of convergence of series related to the reconstruction property in Banach space. First we discuss the uniform convergence of series associated with the reconstruction property in Banach spaces. Necessary and sufficient conditions for the uniform convergence of certain series related to the reconstruction property in Banach spaces are given. A sufficient condition for a Banach space to be finite dimensional in terms of the uniform convergence of a series related to the reconstruction property in Banach spaces is obtained. Motivated by a series of papers by Casazza, we discuss unconditional convergence of series associated with the reconstruction property in Banach spaces. A necessary condition in this direction is given. An absolute type reconstruction property in Banach spaces is also discussed which depends on the absolute convergence of series related to the reconstruction property in Banach spaces.
\end{abstract}

\section{INTRODUCTION}

Let $\mathcal{H}$ be a real (or complex) separable Hilbert space with inner product $\langle.$,$\rangle .$ A sequence $\left\{f_{k}\right\} \subset \mathcal{H}$ is called a frame (or Hilbert frame) for $\mathcal{H}$, if there exist

Date: Received: Mar. 13, 2014; Accepted: Aug. 27, 2014.

* Corresponding author.

2010 Mathematics Subject Classification. Primary 42C15; Secondary 42C05, 42C30, 46 B15.

Key words and phrases. Hilbert space frame, Banach frame, reconstruction property, convergence of series, compact operators, weak*-topology. 
numbers $A, B>0$ such that

$$
A\|f\|^{2} \leq\left\|\left\{\left\langle f, f_{k}\right\rangle\right\}\right\|_{\ell^{2}}^{2} \leq B\|f\|^{2} \text { for all } f \in \mathcal{H} .
$$

The scalars $A$ and $B$ are called lower and upper frame bounds of the frame, respectively. They are not unique. The inequality in (1.1) is called the frame inequality of the frame. The operator $V: \ell^{2} \rightarrow \mathcal{H}$ defined as

$$
V\left(\left\{c_{k}\right\}\right)=\sum_{k=1}^{\infty} c_{k} f_{k},\left\{c_{k}\right\} \in \ell^{2},
$$

is called the pre-frame operator (or synthesis operator) and its adjoint operator $V^{*}: \mathcal{H} \rightarrow \ell^{2}$, which is called the analysis operator is given by

$$
V^{*}(f)=\left\{\left\langle f, f_{k}\right\rangle\right\} \text { for all } f \in \mathcal{H} \text {. }
$$

Composing $V$ and $V^{*}$, we obtain the frame operator $S=V V^{*}: \mathcal{H} \rightarrow \mathcal{H}$ defined by

$$
S(f)=\sum_{k=1}^{\infty}\left\langle f, f_{k}\right\rangle f_{k} \text { for all } f \in \mathcal{H} .
$$

The frame operator $S$ is a positive, self-adjoint and invertible operator on $\mathcal{H}$. This gives the reconstruction formula for all $f \in \mathcal{H}$,

$$
f=S S^{-1} f=\sum_{k=1}^{\infty}\left\langle S^{-1} f, f_{k}\right\rangle f_{k} \quad\left(=\sum_{k=1}^{\infty}\left\langle f, S^{-1} f_{k}\right\rangle f_{k}\right) .
$$

The scalars $\left\{\left\langle S^{-1} f, f_{k}\right\rangle\right\}$ are called frame coefficients of the vector $f \in \mathcal{H}$. The representation of $f$ in the reconstruction formula need not be unique. Today, frames play important roles in many applications in mathematics, science and engineering. In the theoretical direction, powerful tools from the operator theory and Banach spaces are being employed to study frames. An excellent approach towards the utility of frames in different directions in applied mathematics is given in the book by Casazza and Kutyniok [1] and in the paper by Heil and Walnut [22].

Duffin and Schaeffer in [13] while working in nonharmonic Fourier series developed an abstract framework for the idea of time-frequency atomic decomposition by Gabor [19] and defined frames for Hilbert spaces. Due to some reason the theory of frames was not continued until 1986, when the fundamental work of Daubechies, Grossmann and Meyer published in [14]. Gröchenig, generalized Hilbert frames to Banach spaces in [20]. Before the concept of Banach frames was formalized, it appeared in the foundational work of Feichtinger and Gröchenig $[17,18]$ related to the atomic decompositions. Atomic decompositions appeared in the field of applied mathematics providing many applications [2]. An atomic decomposition allow a representation of every vector of the space via a series expansion in terms of a fixed sequence of vectors which we call atoms. On the other hand, Banach frame for a Banach space ensure reconstruction via a bounded linear operator (or synthesis operator). During the development of frames and expansions systems (redundant building blocks), in the later half of twentieth 
century, Coifman and Weiss in [12] introduced the notion of atomic decomposition for function spaces. Later, Feichtinger and Gröchenig extended this idea to Banach spaces. This concept was further generalized by Gröchenig [20], who introduced the notion of Banach frames for Banach spaces. Casazza, Han and Larson discussed a deep analysis on frames and atomic decompositions and Banach frames in [2]. Furthermore, Han and Larson [21] defined a Schauder frame for a Banach space $\mathcal{X}$ to be an inner direct summand (i.e. a compression) of a Schauder basis of $\mathcal{X}$. Since a series is associated with the basis (Schauder) of the underlying space, a natural question about its unconditional convergence arises. This is discussed in a series of papers by Casazza and Casazza et al. (e.g. see $[6,7,9])$.

Recently, the reconstruction property in Banach spaces was introduced and studied by Casazza and Christensen in [11]. The reconstruction property in Banach spaces were further studied in [26, 28, 30]. The reconstruction property is an important tool in several areas of mathematics and engineering. As the perturbation result of Paley and Wiener preserves the reconstruction property, it becomes more important from an application point of view. The reconstruction property is also used to study the geometry of Banach spaces. In fact, it is related to the bounded approximated property (BAP) as observed in [2] and references therein. In [11], Casazza and Christensen gave some perturbation results. In fact, they develop a more general perturbation theory that does not force equivalence of the sequences. Some new results regarding perturbation of the reconstruction property in Banach spaces were discussed in a paper by Kaushik, Khattar and Vashisht [26]. Casazza and Christensen in [11] gave a result which depends on the unconditional convergence of a series which is related to the reconstruction property in Banach spaces. More precisely, they characterize the embedding of the space $c_{0}$ in the dual of a Banach space which has the reconstruction property together with the unconditional convergence of the series related to it. Khattar and Vashisht in [28] gave a type of the reconstruction property in Banach spaces which is generated by the Toeplitz matrices and call it the Toeplitz reconstruction property. They proved in [28] that the standard reconstruction property in a Banach space can generate the Toeplitz reconstruction property from a given Toeplitz matrix but not conversely. Furthermore, sufficient conditions on infinite matrices to have the reconstruction property for a discrete signal space can be found in [28].

1.1. Plan of the work. The convergence (and mode of convergence) of series associated with redundant building blocks is important in applied mathematics. For example, the series associated with frames (reconstruction formula) given in (1.2) is unconditionally convergent, i.e., convergence independent of the ordering of frame vectors. The series associated with the sampling formula [5] converges badly. In particular, it is not absolutely convergent in general. The reconstruction property (which a redundant system) in Banach spaces provides a series expansions of each vector in the underlying space. It would be interesting to know about various mode of convergence of series associated with the reconstruction property in Banach spaces. In this paper, we obtained some new results which 
depends on the mode of convergence of series related to the reconstruction property in Banach spaces. We organize the paper as follows: In Section 2, we give basic definitions and results which will be used throughout the paper. Section 3 deals with the uniform convergence of series associated with the reconstruction property in Banach spaces. Necessary and sufficient conditions for the uniform convergence of certain series related to the reconstruction property in Banach spaces are given. A sufficient condition for a Banach space to be finite dimensional in terms of uniform convergence of a series related to the reconstruction property in Banach spaces is given. Motivated by a series of papers by Casazza, a few important among those are [3, 4, 6, 7, 8, 9], we discuss unconditional convergence of series associated with the reconstruction property in Banach spaces in Section 4. A necessary condition which concerned with the unconditional convergence of series related to the reconstruction property in Banach spaces is obtained. In Section 5, we introduce the notion of the absolute reconstruction property in Banach spaces. Necessary and sufficient conditions concerned with the absolute reconstruction property in Banach spaces are obtained. Construction of Banach frames for a class of compact operators on the underlying space from the reconstruction property are discussed in Section 6 .

\section{Preliminaries}

Throughout this paper $\mathcal{X}$ will denote an infinite dimensional separable real (or complex) Banach space and $\mathcal{X}^{*}$ the conjugate space (topological) of $\mathcal{X}$. For a sequence $\left\{f_{k}\right\} \subset \mathcal{X}$, the closure of linear span of $\left\{f_{k}\right\}$ in the norm topology of $\mathcal{X}$ is denoted by $\left[f_{k}\right]$. The map $\pi: \mathcal{X} \rightarrow \mathcal{X}^{* *}$ denotes the canonical mapping from $\mathcal{X}$ into $\mathcal{X}^{* *}$. The collection of all bounded linear operator from a normed space $X$ into a normed space $Y$ is denoted by $\mathrm{B}(\mathcal{X}, \mathcal{Y})$. By $\mathcal{K}(\mathcal{X})$ we denote the family of all compact linear operator on $\mathcal{X}$. The permutation on $\mathbb{N}=\{1,2,3, \cdots\}$ is denoted by $\rho$. The sign of a scalar (real or complex) denoted by $\operatorname{sign} \alpha$ is defined as $\operatorname{sign} \alpha=\frac{\bar{\alpha}}{|\alpha|}$ for $\alpha \neq 0(\bar{\alpha}$ denote the conjugate of $\alpha)$ and $\operatorname{sign} \alpha=0$ for $\alpha=0$.

Definition 2.1. [20] Let $\mathcal{X}$ be a Banach space and let $\mathcal{X}_{d}$ be an associated Banach space of scalar valued sequences indexed by $\mathbb{N}$. Let $\left\{f_{k}^{*}\right\} \subset \mathcal{X}^{*}$ and $\mathcal{S}: \mathcal{X}_{d} \rightarrow \mathcal{X}$ be given. The pair $\left(\left\{f_{k}^{*}\right\}, \mathcal{S}\right)$ is called a Banach frame for $\mathcal{X}$ with respect to $\mathcal{X}_{d}$, if

(1) $\left\{f_{k}^{*}(f)\right\} \in \mathcal{X}_{d}$ for all $f \in \mathcal{X}$.

(2) There exist numbers $0<A \leq B<\infty$ such that

$$
A\|f\|_{\mathcal{X}} \leq\left\|\left\{f_{k}^{*}(f)\right\}\right\|_{\mathcal{X}_{d}} \leq B\|f\|_{\mathcal{X}} \text { for all } f \in \mathcal{X} .
$$

(3) $\mathcal{S}$ is a bounded linear operator such that

$$
\mathcal{S}\left(\left\{f_{k}^{*}(f)\right\}\right)=f \text { for all } f \in \mathcal{X} .
$$

The scalars $A$ and $B$ are called lower and upper frame bounds of the Banach frame $\left(\left\{f_{k}^{*}\right\}, \mathcal{S}\right)$, respectively. The operator $\mathcal{S}: \mathcal{X}_{d} \rightarrow \mathcal{X}$ is called the reconstruction operator (or pre-frame operator) and the inequality (2.1) is called the frame inequality of the Banach frame. The Banach frame $\left(\left\{f_{k}^{*}\right\}, \mathcal{S}\right)$ is called tight, if 
$A=B$ and normalized tight if, $A=B=1$. If for all $j \in \mathbb{N}$, there is no reconstruction operator $\mathcal{S}_{j}$ such that $\left(\left\{f_{k}\right\}_{k \neq j}, \mathcal{S}_{j}\right)$ is a Banach frame for $\mathcal{X}^{*}$, then $\left(\left\{f_{k}^{*}\right\}, \mathcal{S}\right)$ is called an exact Banach frame for $\mathcal{X}$.

An $F$ space is a topological space if its topology is induced by a complete invariant metric.

Proposition 2.2. [15] (pp. 53) Let $U_{\alpha}$ be a continuous map of an $F$ space $\mathcal{X}_{0}$ into an $F$ space $\mathcal{Y}_{0}$, where $\alpha$ is in a set $A$. If $\left|U_{\alpha}(f+g)\right| \leq\left|U_{\alpha}(f)\right|+\left|U_{\alpha}(g)\right|$ and $\left|U_{\alpha}(t f)\right|=\mid t U_{\alpha}\left(f \mid\right.$ for all $f, g \in \mathcal{X}_{0}$ and for all $t \geq 0$. Then, if for each $f \in \mathcal{X}_{0}$, the set $\left\{U_{\alpha}: \alpha \in A\right\}$ is bounded, the limit $\lim _{f \rightarrow 0} U_{\alpha}(f)=0$ exists uniformly for all $\alpha \in A$.

Proposition 2.3. [16](pp. 27) Let $\left\{f_{n}\right\} \subset \mathcal{X}$ and let $\sum_{n=1}^{\infty} f_{n}$ be a series of vectors in $\mathcal{X}$. Then, the following are equivalent.

(1) If $\rho($.$) is any permutation of \mathbb{N} ; \sum_{n=1}^{\infty} f_{\rho(n)}=f, f \in \mathcal{X}$.

(2) For each $\epsilon>0$, there is a finite set $\Omega \subset \mathbb{N}$ such that $\left\|f-\sum_{j \in \Omega_{0}} f_{j}\right\|<\epsilon$, whenever $\Omega_{0} \subset \mathbb{N}$ is finite set such that $\Omega_{0} \supset \Omega$.

Definition 2.4. [11] Let $\mathcal{X}$ be a separable Banach space . A sequence $\left\{f_{k}^{*}\right\} \subset \mathcal{X}^{*}$ has the reconstruction property for $\mathcal{X}$ with respect to a sequence $\left\{f_{k}\right\} \subset \mathcal{X}$ if

$$
f=\sum_{k=1}^{\infty} f_{k}^{*}(f) f_{k} \text { for all } f \in \mathcal{X} .
$$

In short, we will also say that the pair $\left(\left\{f_{k}\right\},\left\{f_{k}^{*}\right\}\right)$ has the reconstruction property for $\mathcal{X}$. More precisely, we say that $\left(\left\{f_{k}\right\},\left\{f_{k}^{*}\right\}\right)$ is a reconstruction system for $\mathcal{X}$.

Remark 2.5. An interesting example for the reconstruction property is given in [11]: Let $\left\{f_{k}^{*}\right\} \subset \ell^{\infty}$ and $\left\{f_{k}^{*}\right\}$ is unitarily equivalent to the unit vector basis of $\ell^{2}$. Then, $\left\{f_{k}^{*}\right\}$ has the reconstruction property with respect to its own predual (that is, expansions with respect to the orthonormal basis). But this family cannot have the reconstruction property with respect to $\ell^{1}$, which is the pre-dual of $\ell^{\infty}$.

Definition 2.6. [2] A separable Banach space $\mathcal{X}$ has the $\lambda$-Bounded Approximation Property (i.e. $\lambda$-BAP), if there is a sequence of finite rank operators $\left\{T_{i}\right\}$ defined on $\mathcal{X}$ such that $\left\|T_{i}\right\| \leq \lambda$ for all $i$ and for every $f \in \mathcal{X}, T_{i} f \rightarrow f$ in norm. We say that $\mathcal{X}$ has the Bounded Approximation Property (denoted by BAP) if $\mathcal{X}$ has the $\lambda$-BAP, for some $\lambda$.

The notion of the reconstruction property in Banach spaces is related to the Bounded Approximation Property(BAP). If $\left.\left\{f_{k}^{*}\right\}\right)$ has the reconstruction property for $\mathcal{X}$ with respect to $\left(\left\{f_{k}\right\}\right.$, then $\mathcal{X}$ has the bounded approximation property. Conversely, if $\mathcal{X}$ has the bounded approximation property then there exists a Banach space $\mathcal{A} \supset \mathcal{X}$ with a basis and by using a projection $P: \mathcal{A} \rightarrow \mathcal{X}$ we can find a sequence $\left\{g_{k}^{*}\right\} \subset \mathcal{X}^{*}$ such that $\left\{g_{k}^{*}\right\}$ has reconstruction property for $\mathcal{X}$ with 
respect to $\{P(\bullet)\}_{k}$. So, $\mathcal{X}$ is isomorphic to a complemented subspace of a Banach space with a basis. The reconstruction property is also used to study geometry of Banach spaces [2]. For more results on the bounded approximation property one may refer to [2] and references therein. An interested reader for approximation property in Banach spaces may refer to [10, 23, 24].

Definition 2.7. Suppose that $\left\{f_{k}^{*}\right\}$ has the reconstruction property for $\mathcal{X}$ with respect to $\left\{f_{k}\right\}$. Then, $\left(\left\{f_{k}\right\},\left\{f_{k}^{*}\right\}\right)$ is said to be

(1) pre-shrinking, if $\left[f_{k}^{*}\right]=\mathcal{X}^{*}$.

(2) shrinking, if $\left(\left\{f_{k}^{*}\right\},\left\{f_{k}\right\}\right)$ has the reconstruction property for $\mathcal{X}^{*}$.

Regarding the existence of Banach spaces which have a reconstruction system, Casazza and Christensen proved the following result.

Proposition 2.8. [11] There exists a Banach space $\mathcal{X}$ with the following properties:

(1) There is a sequence $\left\{f_{k}\right\}$ such that each $f \in \mathcal{X}$ has a expansion $f=$ $\sum_{k=1}^{\infty} a_{k} f_{k}$.

(2) $\mathcal{X}$ does not have the reconstruction property with respect to any pair $\left(\left\{h_{k}\right\},\left\{h_{k}^{*}\right\}\right)$.

\section{Uniform Convergence Related to the Reconstruction Property in Banach Spaces}

In this section, we discuss uniform convergence of series related to the reconstruction property in Banach spaces. First we discuss the uniform convergence of a series associated with the reconstruction property on bounded subsets of the underlying space. In this direction the following theorem gives a necessary and sufficient conditions for the uniform convergence of a certain series related to the reconstruction property in Banach spaces.

Theorem 3.1. Assume that $\left\{f_{k}^{*}\right\}$ has the reconstruction property for $\mathcal{X}$ with respect to $\left\{f_{k}\right\}$. Then, the series

$$
\sum_{k=1}^{\infty} f_{k}^{*}(f) f^{*}\left(f_{k}\right)
$$

is uniformly convergent on each bounded set $M \subset \mathcal{X}$, for all $f^{*} \in \mathcal{X}^{*}$ if and only if

$$
\lim _{n \rightarrow \infty} \sup _{f \in M \cap \mathcal{X}^{n}}\left|f^{*}(f)\right|=0,
$$

where $\mathcal{X}^{n}=f_{n+1} \oplus f_{n+2} \oplus \ldots .(n \in \mathbb{N})$.

Proof. Assume first that the series given in (3.1) is uniformly convergent on each bounded set $M \subset \mathcal{X}$, for all $f^{*} \in \mathcal{X}^{*}$. Let $\epsilon>0$. Then, by hypothesis, we can choose a positive integer $n_{\epsilon}$ such that for $n \geq n_{\epsilon}$ and that

$$
\sup _{f \in M}\left|\sum_{k=n+1}^{\infty} f_{k}^{*}(f) f^{*}\left(f_{k}\right)\right|<\epsilon .
$$


Now

$$
\sup _{f \in M \cap \mathcal{X}^{n}}\left|f^{*}(f)\right|=\sup _{f \in M}\left|\sum_{k=n+1}^{\infty} f_{k}^{*}(f) f^{*}\left(f_{k}\right)\right|<\epsilon .
$$

Therefore, for every bounded set $M \subset \mathcal{X}$, we have

$$
\lim _{n \rightarrow \infty} \sup _{f \in M \cap \mathcal{X}^{n}}\left|f^{*}(f)\right|=0 \text {. }
$$

For the reverse part, let $f^{*} \in \mathcal{X}^{*}$ be arbitrary and assume that

$$
\lim _{n \rightarrow \infty} \sup _{f \in M \cap \mathcal{X}^{n}}\left|f^{*}(f)\right|=0 \text {. }
$$

Define

$$
R_{n}(f)=\sum_{k=n+1}^{\infty} f_{k}^{*}(f) f_{k}, f \in \mathcal{X}(n \in \mathbb{N}) .
$$

Then, each $R_{n}$ is a continuous linear operator on $\mathcal{X}$. By using the BanachSteinhaus Theorem, we have

$$
\sup _{1 \leq n \leq \infty}\left\|R_{n}\right\|<\infty
$$

It follows that for any bounded set $M$ in $\mathcal{X}$, there is a bounded set $\mathcal{Q} \subset \mathcal{X}$ such that $R_{n}(M) \subseteq \mathcal{Q}$. This gives $R_{n}(M) \subseteq \mathcal{Q} \cap \mathcal{X}^{n}$. Therefore, by using (3.2), for each $\epsilon>0$, there exists a positive integer $n_{\epsilon}$ such that

$$
\sup _{f \in \mathcal{Q} \cap \mathcal{X}^{n}}\left|f^{*}(f)\right|<\epsilon \text { for all } n \geq n_{\epsilon} \text {. }
$$

We compute

$$
\begin{aligned}
\sup _{f \in M}\left|\sum_{k=n+1}^{\infty} f_{k}^{*}(f) f^{*}\left(f_{k}\right)\right| & =\sup _{f \in M}\left|f^{*}\left(R_{n}(f)\right)\right| \\
& =\sup _{g \in R_{n}(M)}\left|f^{*}(g)\right| \\
& \leq \sup _{g \in \mathcal{Q} \cap \mathcal{X}^{n}}\left|f^{*}(g)\right| \\
& <\epsilon .
\end{aligned}
$$

Therefore, $\sum_{k=1}^{\infty} f_{k}^{*}(f) f^{*}\left(f_{k}\right)$ is uniformly convergent on each bounded set $M \subset \mathcal{X}$ for all $f^{*} \in \mathcal{X}^{*}$.

The following proposition gives a type of representation of the conjugate $T^{*}$ of the operator $T$ which is associated with the uniform convergence of a series related to the reconstruction property in Banach spaces.

Proposition 3.2. Suppose that $\left\{f_{k}^{*}\right\}$ has the reconstruction property for a Banach space $\mathcal{X}$ with respect to $\left\{f_{k}\right\}$ and let $\left\{g_{k}\right\} \subset \mathcal{X}$. Let $T \in \mathrm{B}\left(\mathcal{X}^{*}, \mathcal{X}^{*}\right)$ be given by

$$
T\left(f^{*}\right)=\sum_{k=1}^{\infty} f^{*}\left(f_{k}-g_{k}\right) f_{k}^{*}
$$


and let $\sum_{k=1}^{\infty} f^{*}\left(f_{k}-g_{k}\right) f_{k}^{*}$ converges uniformly on the unit ball $S_{\mathcal{X}^{*}} \subset \mathcal{X}^{*}$. Then

$$
T^{*} \pi_{f}=\sum_{k=1}^{\infty} f_{k}^{*}(f)\left(f_{k}-g_{k}\right)
$$

where $\pi_{f}$ is given by $\pi_{f}\left(f^{*}\right)=f^{*}(f)$ for all $f^{*} \in \mathcal{X}^{*}$.

Proof. By using uniform convergence of the series $\sum_{k=1}^{\infty} f^{*}\left(f_{k}-g_{k}\right) f_{k}^{*}$ on $S_{\mathcal{X}^{*}}$, we have

$$
\sup _{f^{*} \in S_{\mathcal{X}^{*}}}\left\|\sum_{k=m}^{n} f^{*}\left(f_{k}-g_{k}\right) f_{k}^{*}\right\| \rightarrow 0 \text { as } m, n \rightarrow \infty .
$$

By using (3.3), we conclude that $\sum_{k=1}^{\infty} f_{k}^{*}(f)\left(f_{k}-g_{k}\right)$ converges for each $f \in \mathcal{X}$. By using the Banach-Steinhaus Theorem, the operator $\Theta: \mathcal{X} \rightarrow \mathcal{X}$ given by

$$
\Theta(f)=\sum_{k=1}^{\infty} f_{k}^{*}(f)\left(f_{k}-g_{k}\right)
$$

is continuous.

We compute

$$
\begin{aligned}
T^{*} \pi_{(f)}\left(f^{*}\right) & =\left(\pi_{f}\right) T\left(f^{*}\right) \\
& =T\left(f^{*}(f)\right) \\
& =\sum_{k=1}^{\infty} f^{*}\left(f_{k}-g_{k}\right) f_{k}^{*}(f) \\
& =\sum_{k=1}^{\infty} f_{k}^{*}(f)\left(f_{k}-g_{k}\right)\left(f^{*}\right), f^{*} \in \mathcal{X}^{*} .
\end{aligned}
$$

The proposition is proved.

3.1. The reconstruction property in finite dimensional Banach spaces. Now we discuss the reconstruction property in finite dimensional Banach spaces. It is well known that a finite dimensional Banach space has the reconstruction property indexed by a finite set. Furthermore, we can construct an infinite sequence $\left\{f_{n}^{*}\right\}$ in the conjugate space of a given finite dimensional Banach which admits the reconstruction property with respect to some $\left\{f_{n}\right\}$ in the underlying space. More precisely, let $\mathfrak{X}$ be a real (or complex) finite dimensional Banach space. Then, we can construct an infinite sequence $\left\{f_{n}^{*}\right\} \subset \mathfrak{X}^{*}$ such that $\left\{f_{n}^{*}\right\}$ has the reconstruction property for $\mathcal{X}$ with respect to some $\left\{f_{n}\right\} \subset \mathfrak{X}$. Indeed, if $\operatorname{dim} \mathfrak{X}<\infty$, then there exist sequences $\left\{h_{i}\right\}_{i=1}^{n} \subset \mathfrak{X}$ and $\left\{h_{i}^{*}\right\}_{i=1}^{n} \subset \mathfrak{X}^{*}$ such that

$$
f=\sum_{i=1}^{n} h_{i}^{*}(f) h_{i} \text { for all } f \in \mathfrak{X} .
$$


Now we define two infinite sequences $\left\{f_{n}\right\} \subset \mathfrak{X}$ and $\left\{f_{n}^{*}\right\} \subset \mathfrak{X}^{*}$ as follows:

$$
\left.\begin{array}{rl}
f_{t n^{2}+l n+\xi} & =\frac{1}{2^{t+1} n} h_{\xi} \\
f_{t n^{2}+l n+\xi}^{*} & =h_{\xi}^{*}
\end{array}\right\} \quad\left(\begin{array}{c}
t=0,1,2, \ldots ; \\
l=0,1, \ldots, n-1 ; \xi=1,2, \ldots, n
\end{array}\right)
$$

Let $f \in \mathfrak{X}$ be arbitrary. Then, by using (3.4), we have

$$
\begin{aligned}
\sum_{i=1}^{\infty} f_{i}^{*}(f) f_{i} & =\sum_{t=0}^{\infty} \sum_{l=0}^{n-1} \sum_{\xi=1}^{n} f_{t n^{2}+l n+\xi}^{*}(f) f_{t n^{2}+l n+\xi} \\
& =\sum_{t=0}^{\infty} n \sum_{\xi=1}^{n} \frac{1}{2^{t+1} n} h_{\xi}^{*}(f) h_{\xi} \\
& =\sum_{\xi=1}^{n} h_{\xi}^{*}(f) h_{\xi} \\
& =f .
\end{aligned}
$$

Hence $\left\{f_{n}^{*}\right\} \subset \mathfrak{X}^{*}$ has the reconstruction property (infinite) for $\mathfrak{X}$ with respect to $\left\{f_{n}\right\}$.

On the other hand, if $\mathcal{X}$ is a Banach space which have the reconstruction property, then dimension of $\mathcal{X}$ need not be finite. It would be interesting to know what condition(s) we should impose on the reconstruction property for the Banach space $\mathcal{X}$, so that dimension of $\mathcal{X}$ be finite. The answer in this direction is based upon the uniform convergence of the certain series associated with the reconstruction property for the underlying space. More precisely, if a certain series related to the reconstructed property (for $\mathcal{X}$ ) converge uniformly on the unit ball of the Banach space $\mathcal{X}$, then dimension of $\mathcal{X}$ is finite. The following lemma provides a sufficient condition for the dimension of Banach spaces (which possesses the reconstruction property) to be finite.

Lemma 3.3. Assume that $\left\{f_{k}^{*}\right\}$ has the reconstruction property for $\mathcal{X}$ with respect to $\left\{f_{k}\right\}$ and with the property that the series $\sum_{k=1}^{\infty} f_{k}^{*}(f) f_{k}$ converges uniformly on the unit ball $S_{\mathcal{X}}=\{f \in \mathcal{X}:\|f\| \leq 1\}$. Then, dimension of $\mathcal{X}$ is finite.

Proof. For each $n \in \mathbb{N}$, define $\Theta_{n}: \mathcal{X} \rightarrow \mathcal{X}$ by

$$
\Theta_{n}(f)=\sum_{k=1}^{n} f_{k}^{*}(f) f_{k}, f \in \mathcal{X}
$$

Let $I: \mathcal{X} \rightarrow \mathcal{X}$ be the identity operator.

We compute

$$
\begin{aligned}
\left\|I-\Theta_{n}\right\| & =\sup _{\substack{f \in \mathfrak{X} \\
\|f\| \leq 1}}\left\|f-\sum_{k=1}^{n} f_{i}^{*}(f) f_{k}\right\| \\
& =\sup _{\substack{f \in \mathfrak{x} \\
\| f \leq 1}}\left\|\sum_{k=n+1}^{\infty} f_{k}^{*}(f) f_{k}\right\|
\end{aligned}
$$




$$
\rightarrow 0 \text { as } n \rightarrow \infty \text {. }
$$

Now, $\operatorname{dim} \Theta_{n}(\mathcal{X})=\operatorname{dim}\left[f_{k}\right]_{k=1}^{n}$, which is finite for all $n \in \mathbb{N}$. Thus, each $\Theta_{n}$ is compact, so $I$ is compact. Therefore, the unit ball $S_{\mathcal{X}}=\mathrm{I}\left(S_{\mathcal{X}}\right)$ is compact. Hence dimension of $\mathcal{X}$ is finite.

Corollary 3.4. Suppose that $\left\{f_{k}^{*}\right\}$ has the reconstruction property for the infinite dimensional Banach space $\mathcal{X}$ with respect to $\left\{f_{k}\right\}$. Then, the series $\sum_{k=1}^{\infty} f_{k}^{*}(f) f_{k}$ never converges uniformly on the unit ball $S_{\mathcal{X}}$ of the Banach space $\mathcal{X}$.

Remark 3.5. Karlin in [27], first observed the result given in Lemma 3.3, in the context of uniform bases in Banach spaces. Here we generalize it to the reconstruction property in Banach spaces.

3.2. The reconstruction property and convergence in the $\omega^{*}$-topology. Casazza and Christensen gave a result in [11] regarding convergence of series associated with the reconstruction property in the $\omega^{*}$-topology. For completeness we include its proof.

Proposition 3.6. [11] Suppose that $\left(\left\{f_{k}\right\},\left\{f_{k}^{*}\right\}\right)$ has the reconstruction property for a Banach space $\mathcal{X}$. Then, for all $g \in \mathcal{X}^{*}$, we have that the sequence

$$
\left\{\sum_{k=1}^{n} g\left(f_{k}\right) f_{k}^{*}\right\}
$$

converges to $g \in \mathcal{X}^{*}$ in the $\omega^{*}$-topology.

Proof. Let $f \in \mathcal{X}$ be arbitrary. Then,

$$
\begin{aligned}
\lim _{n \rightarrow \infty}\left[\left\{\sum_{k=1}^{n} g\left(f_{k}\right) f_{k}^{*}\right\}(f)\right] & =\lim _{n \rightarrow \infty} \sum_{k=1}^{n} g\left(f_{k}\right) f_{k}^{*}(f) \\
& =\lim _{n \rightarrow \infty} g\left(\sum_{k=1}^{n} f_{k}^{*}(f) f_{k}\right) \\
& =g\left(\lim _{n \rightarrow \infty} \sum_{k=1}^{n} f_{k}^{*}(f) f_{k}\right) \\
& =g(f) .
\end{aligned}
$$

The proposition is proved.

Let us have a look at the weak*-convergence of the sequence given in (3.5). For all $g \in \mathcal{X}^{*}$, we have

$$
\begin{aligned}
g & =w^{*}-\lim _{n \rightarrow \infty} \sum_{k=1}^{n} g\left(f_{k}\right) f_{k}^{*} \\
& =w^{*}-\lim _{n \rightarrow \infty} \sum_{k=1}^{n}\left(\Psi_{k}^{* *}\right)(g) f_{k}^{*},
\end{aligned}
$$


where $\Psi_{k}^{* *} \equiv \pi_{f_{k}}$ for all $k \in \mathbb{N}$. That is, all the coefficient functionals appearing in the sequence (3.5) are weak*-continuous. This is not true, in general, for the reconstruction property for the conjugate space of the underlying space. More precisely, assume that $\left\{\Psi_{k}^{* *}\right\}$ has the reconstruction property for $\mathcal{X}^{*}$ with respect to $\left\{f_{k}^{*}\right\}$. Then, in general, the sequence $\left\{\Psi_{k}^{* *}\right\}$ is not weak ${ }^{*}$-continuous. That is, there is no sequence $\left\{f_{k}\right\} \subset \mathcal{X}$ such that $\pi_{f_{k}}=\Psi_{k}^{* *}$ for all $k \in \mathbb{N}$. This is discussed in the following example.

Example 3.7. Let $\mathcal{X}=\left(c_{0},\|\cdot\|_{\infty}\right)$ and let $\chi_{k}=\{0,0, \cdots, \underbrace{1}_{k t h}, 0,0,0, \cdots\}, k \in$ $\mathbb{N}$. Consider a sequence $\left\{\Psi_{k}^{* *}\right\} \subset \mathcal{X}^{* *}$ which is defined as follows:

$$
\Psi_{1}^{* *}\left(f^{*}\right)=\xi_{1}+\sum_{j=2}^{\infty}(-1)^{j} \xi_{j}, f^{*}=\left\{\xi_{j}\right\} \subset \mathcal{X}^{*},
$$

and

$$
\Psi_{k}^{* *}\left(f^{*}\right)=\xi_{k}, k \in \mathbb{N} \backslash\{1\}, f^{*}=\left\{\xi_{j}\right\} \subset \mathcal{X}^{*} .
$$

Then, $\left\{\Psi_{k}^{* *}\right\}$ has the reconstruction property for $\mathcal{X}^{*}$ with respect to $\left\{f_{k}^{*}\right\}$, where

$$
f_{1}^{*}=\chi_{1}, f_{k}^{*}=(-1)^{k+1} \chi_{1}+\chi_{k}, k \in \mathbb{N} \backslash\{1\} .
$$

Indeed, let $f=\left\{\xi_{j}\right\} \in \mathcal{X}^{*}$ be arbitrary. Then, for all $n \in \mathbb{N}$, we have

$$
\begin{aligned}
\sum_{j=1}^{n} \Psi_{j}^{* *}\left(f^{*}\right) f_{j}^{*} & =\left(\xi_{1}+\sum_{j=2}^{\infty}(-1)^{j} \xi_{j}\right) \chi_{1}+\sum_{j=2}^{n} \xi_{j}\left((-1)^{j+1} \chi_{1}+\chi_{j}\right) \\
& =\sum_{j=1}^{n} \xi_{j} \chi_{j}+\left(\sum_{j=n+1}^{\infty}(-1)^{j} \xi_{j}\right) \chi_{1} .
\end{aligned}
$$

Since $f^{*}=\left\{\xi_{j}\right\} \in \mathcal{X}^{*}$, by using (3.6) we have

$$
f^{*}=\sum_{k=1}^{\infty} \Psi_{k}^{* *}\left(f^{*}\right) f_{k}^{*}
$$

Therefore, $\left\{\Psi_{k}^{* *}\right\}$ has the reconstruction property for $\mathcal{X}^{*}$ with respect to $\left\{f_{k}^{*}\right\}$. But the system of coefficient functionals appearing in (3.7) is not weak*-continuous. The reason is that $\Psi_{1}^{* *}$ is not in $\pi(\mathcal{X})$.

\section{Unconditional Convergence Related to the Reconstruction Property in Banach Spaces}

Definition 4.1. Suppose that $\left\{f_{k}^{*}\right\}$ has the reconstruction property for $\mathcal{X}$ with respect to $\left\{f_{k}\right\}$. We say that $\left(\left\{f_{k}\right\},\left\{f_{k}^{*}\right\}\right)$ is unconditional, if the series

$$
\sum_{k=1}^{\infty} f_{k}^{*}(f) f_{k}
$$

is unconditionally convergent for all $f \in \mathcal{X}$. That is, if $\sum_{k=1}^{\infty} f_{\rho(k)}^{*}(f) f_{\rho(k)}$ converges for all $f \in \mathcal{X}$, where $\rho($.$) is any permutation of \mathbb{N}$. 
Example 4.2. Let $\mathcal{X}=L^{1}\left(\Omega_{o}, \mu\right)$, where $\Omega_{o}=\mathbb{N}$ and $\mu$ is the counting measure and let $\chi_{k}=\{0,0, \cdots, \underbrace{1}_{k t h}, 0,0,0, \cdots\}(k \in \mathbb{N})$.

Define $\left\{f_{k}^{*}\right\} \subset \mathcal{X}^{*}$ by

$$
f_{1}^{*}(f)=f_{2}^{*}(f)=\frac{\xi_{1}}{2}, f=\left\{\xi_{j}\right\} \in \mathcal{X}
$$

and

$$
f_{k}^{*}(f)=\xi_{k-1}, k>2, f=\left\{\xi_{j}\right\} \in \mathcal{X}
$$

Choose $f_{1}=f_{2}=\chi_{1}$ and $f_{k}=\chi_{k-1}, k>2$. Then, $\left\{f_{k}^{*}\right\}$ has the reconstruction property for $\mathcal{X}$ with respect to $\left\{f_{k}\right\}$ which is unconditional.

Indeed, let $f=\left\{\xi_{j}\right\} \in \mathcal{X}$ be arbitrary. Then, for $n, p \in \mathbb{N}$, we have

$$
\left\|\sum_{k=n}^{n+p} f_{k}^{*}(f) f_{k}\right\|=\sum_{k=n}^{n+p}\left|\xi_{k}\right| .
$$

Now the series $\sum_{k=1}^{\infty}\left|\xi_{k}\right|$ converges unconditionally, so is $\sum_{k=1}^{\infty} f_{k}^{*}(f) f_{k}$. Hence $\left\{f_{k}^{*}\right\}$ has the unconditional reconstruction property for $\mathcal{X}$ with respect to $\left(\left\{f_{k}\right\}\right.$.

Example 4.3. Consider the Banach space $\mathcal{X}=\left(c_{0},\|\cdot\|_{\infty}\right)$.

Define $\left\{f_{k}^{*}\right\} \subset \mathcal{X}^{*}$ by

$$
f_{k}^{*}(f)=\left\{0,0,0, \cdots, \xi_{k},-\xi_{k+1}, 0,0, \cdots\right\}, f=\left\{\xi_{j}\right\} \in \mathcal{X}(k \in \mathbb{N}) .
$$

For each $k \in \mathbb{N}$, choose $f_{k}=\sum_{i=1}^{k} \chi_{k}$, where $\left\{\chi_{k}\right\}$ is the sequence of canonical unit vector given in Example 4.2. Then, $\left\{f_{k}^{*}\right\}$ has the reconstruction property for $\mathcal{X}$ with respect to $\left\{f_{k}\right\}$. To show $\left(\left\{f_{n}\right\},\left\{f_{n}^{*}\right\}\right)$ is not unconditional. Let $f \in \mathcal{X}$ be arbitrary. Then, for $n, p \in \mathbb{N}$, we have

$$
\left\|\sum_{k=n}^{n+p} f_{k}^{*}(f) f_{k}\right\|_{\infty}=\sup _{n \leq l \leq n+p}\left|\sum_{k=l}^{n+p} f_{k}^{*}(f)\right| .
$$

Choose $f_{0}=\left\{0, \frac{1}{2}, 0, \frac{1}{3}, ..\right\} \in \mathcal{X}$. Then, for this $f_{0}$, the series $\sum_{i=1}^{\infty} f_{i}^{*}\left(f_{0}\right)$ is conditionally convergent. Therefore, the reconstruction system $\left(\left\{f_{n}\right\},\left\{f_{n}^{*}\right\}\right)$ is not unconditional.

Casazza and Christensen proved in [11] that we can get the reconstruction property with respect to $\mathcal{X}^{*}$, if the reconstruction property for $\mathcal{X}$ holds with unconditional convergence. More precisely, they characterize the embedding of the space $c_{0}$ in the dual of a Banach space $\mathcal{X}$ which has the reconstruction property together with the unconditional convergence of the series $\sum_{k=1}^{\infty} f_{k}^{*}(f) f_{k}$ for all $f \in \mathcal{X}$.

Proposition 4.4. [11] Assume that $\left(\left\{f_{k}\right\},\left\{f_{k}^{*}\right\}\right)$ has the reconstruction property for $\mathcal{X}$ and that the series $\sum_{k=1}^{\infty} f_{k}^{*}(f) f_{k}$ converges unconditionally for all $f \in \mathcal{X}$. Then, the following are equivalent. 
(1) For all $g \in \mathcal{X}^{*}$, we have

$$
g=\sum_{k=1}^{\infty} g\left(f_{k}\right) f_{k}^{*}
$$

(2) $c_{o}$ does not embed into $\mathcal{X}^{*}$.

We now discuss necessary conditions for the reconstruction property to be unconditional. First we give a necessary condition which is related to the convergence of a particular type of the series, derived from the unconditional reconstruction property. More precisely, assume that $\left\{f_{k}^{*}\right\}$ has the unconditional reconstruction property for $\mathcal{X}$ with respect to $\left\{f_{k}\right\}$ and let $\left\{\xi_{k}\right\}$ be a sequence of scalars. It is difficult to say about the convergence of the series $\left(\mathcal{W}_{k=1}^{\infty}\left|\xi_{k} f^{*}\left(f_{k}\right)\right|\right.$ $\left(f^{*} \in \mathcal{X}^{*}\right)$. A special type of sequence of scalars can be generated by the action of $\left\{f_{k}^{*}\right\}$ on a certain sequence in $\mathcal{X}$, so that the series ( converges for all $f^{*} \in \mathcal{X}^{*}$. The following theorem gives a necessary condition in this direction for the unconditional reconstruction property in Banach spaces.

Theorem 4.5. Assume that $\left\{f_{k}^{*}\right\}$ has the unconditional reconstruction property for $\mathcal{X}$ with respect to $\left\{f_{k}\right\}$. Let $\left\{g_{n}\right\} \subset \mathcal{X}$ be a sequence such that $\sup _{1 \leq n \leq \infty}\left\|g_{n}\right\|<\infty$ and let $\left\{\xi_{k}\right\}$ be a sequence of scalars given by $\xi_{k}=\lim _{n \rightarrow \infty} f_{k}^{*}\left(g_{n}\right)(k \in \mathbb{N})$. Then, the series

$$
\sum_{k=1}^{\infty}\left|\xi_{k} f^{*}\left(f_{k}\right)\right|
$$

converges for all $f^{*} \in \mathcal{X}^{*}$.

Proof. Let $\Theta_{n, \epsilon_{i}}=\sum_{i=1}^{n} \epsilon_{i} f^{*}\left(f_{i}\right) f_{i}^{*}$, where $\epsilon_{i}= \pm 1(i=1,2 \cdots, n), n \in \mathbb{N}$. First we show that

$$
(\star) \equiv\left\|\Theta_{n, \epsilon_{i}}\right\| \leq C,(i=1,2 \cdots, n), n \in \mathbb{N} .
$$

Define

$$
U_{n}(f)=\sum_{i=1}^{n}\left|f^{*}\left(f_{i}\right) f_{i}^{*}(f)\right|, f \in \mathcal{X}(n \in \mathbb{N}) .
$$

Then, for all $f, g \in \mathcal{X}$, we have

$$
\begin{aligned}
\left|U_{n}(f+g)\right| & =\left|\sum_{i=1}^{n}\right| f^{*}\left(f_{i}\right) f_{i}^{*}(f+g)|| \\
& \leq\left|U_{n}(f)\right|+\left|U_{n}(g)\right|, n \in \mathbb{N} .
\end{aligned}
$$

Similarly for all $\alpha \in \mathbb{K}, \alpha \geq 0$ and for all $f \in \mathcal{X}$, we can show that

$$
\left|\alpha U_{n}(f)\right|=\left|U_{n}(\alpha f)\right|, n \in \mathbb{N} .
$$

Now $\left\{f_{k}^{*}\right\}$ has the unconditional reconstruction property for $\mathcal{X}$ with respect to $\left\{f_{k}\right\}$. Therefore, for every $f^{*} \in \mathcal{X}^{*}$, the series $\sum_{k=1}^{\infty} f^{*}\left(f_{k}\right) f_{k}^{*}$ is $\sigma\left(\mathcal{X}^{*}, \mathcal{X}\right)$ is unconditionally convergent to $f^{*}$. Thus, for each $f \in \mathcal{X},\left\{U_{n}(f): n \in \mathbb{N}\right\}$ is bounded. Therefore, by Proposition 2.2, $\lim _{f \rightarrow 0} U_{n} f=0$ exists uniformly in $\mathcal{X}$ for all $n \in \mathbb{N}$. Thus, there exists a constant $C>0$ such that 


$$
\sum_{i=1}^{n}\left|f^{*}\left(f_{i}\right) f_{i}^{*}(f)\right| \leq C\|f\| \text { for all } f \in \mathcal{X} \text { and for all } n \in \mathbb{N} \text {. }
$$

Since

$$
\begin{aligned}
\left\|\Theta_{n, \epsilon_{i}}\right\| & =\left\|\sum_{i=1}^{n} \zeta_{i} f^{*}\left(f_{i}\right) f_{i}^{*}-\sum_{i=1}^{n} \zeta_{i}^{\prime} f^{*}\left(f_{i}\right) f_{i}^{*}\right\| \\
& \leq\left\|\sum_{i=1}^{n} \zeta_{i} f^{*}\left(f_{i}\right) f_{i}^{*}\right\|+\left\|\sum_{i=1}^{n} \zeta_{i}^{\prime} f^{*}\left(f_{i}\right) f_{i}^{*}\right\| .
\end{aligned}
$$

where $\zeta_{i}=1, \zeta_{i}^{\prime}=0$ if $\epsilon_{i}=1$ and $\zeta_{i}=0, \zeta_{i}^{\prime}=1$ if $\epsilon_{i}=-1(i=1,2,3 \cdots n), n \in \mathbb{N}$. Therefore, by using (4.2) in (4.3), we obtain

$$
\left\|\Theta_{n, \epsilon_{i}}\right\| \leq C(i=1,2 \cdots, n), n \in \mathbb{N}
$$

Thus, $(\star)$ is proved.

For arbitrary $f^{*} \in \mathcal{X}^{*}$, by using $(\star)$, we have

$$
\begin{aligned}
\sum_{i=1}^{n}\left|\xi_{i} f^{*}\left(f_{i}\right)\right| & =\sum_{i=1}^{n}\left|f^{*}\left(\epsilon_{i} \xi_{i} f_{i}\right)\right| \\
& =\sum_{i=1}^{n}\left(\operatorname{sign} f^{*}\left(f_{i}\right) \xi_{i}\right) f^{*}\left(f_{i}\right) \xi_{i} \\
& =\lim _{k \rightarrow \infty} \sum_{i=1}^{n}\left(\operatorname{sign} f^{*}\left(f_{i}\right) \xi_{i}\right) f^{*}\left(f_{i}\right) f_{i}^{*}\left(g_{k}\right) \\
& \leq \varlimsup_{k \rightarrow \infty}\left\|\sum_{i=1}^{n}\left(\operatorname{sign} f^{*}\left(f_{i}\right) \alpha_{i}\right) f^{*}\left(f_{i}\right) f_{i}^{*}\right\|\left\|g_{k}\right\| \\
& \leq\left\|\sum_{i=1}^{n}\left(\operatorname{sign} f^{*}\left(f_{i}\right) \xi_{i}\right) f^{*}\left(f_{i}\right) f_{i}^{*}\right\|\left(\sup _{1 \leq k \leq \infty}\left\|g_{k}\right\|\right) \\
& \leq C \sup _{1 \leq k \leq \infty}\left\|g_{k}\right\| \\
& <\infty .
\end{aligned}
$$

Therefore, for all $f^{*} \in \mathcal{X}^{*}$, the series $\sum_{k=1}^{\infty}\left|\xi_{k} f^{*}\left(f_{k}\right)\right|$ (given in (4.1)) is convergent. The theorem is proved.

The convergence of a series related to the frames or the reconstruction system on the unit ball of the underlying space is always interesting. The following theorem provides a necessary condition for the reconstruction property for $\mathcal{X}$ to be unconditional in terms of the convergence of a certain sequence generated by the given reconstruction system, over the unit ball of $\mathcal{X}^{*}$. We use certain ideas developed in $[6,9,16,25,29]$. 
Theorem 4.6. Assume that $\left\{f_{k}^{*}\right\}$ has the unconditional reconstruction property for $\mathcal{X}$ with respect to $\left\{f_{k}\right\}$. Then, for every $f \in \mathcal{X}$

$$
\lim _{n \rightarrow \infty} \sup _{f^{*} \in S_{\mathcal{X}^{*}}} \sum_{i=n+1}^{\infty}\left|f^{*}\left(f_{i}\right)\right|\left|f_{i}^{*}(f)\right|=0
$$

Proof. Let $\epsilon>0$ be given. Since $\left\{f_{n}^{*}\right\}$ has the unconditional reconstruction property for $\mathcal{X}$ with respect to $\left\{f_{n}\right\}$. By Proposition 2.3, there exists a finite subset $\Omega$ of $\mathbb{N}$ such that

$$
\left\|f-\sum_{i \in \Omega^{\prime}} f_{i}^{*}(f) f_{i}\right\|<\frac{\epsilon}{4}, \text { for all finite subsets } \Omega^{\prime} \subset \mathbb{N}\left(\Omega^{\prime} \subset \Omega\right) .
$$

Define sets

$$
\Omega_{1}(f)=\left\{i \in\{n+1, n+2, \cdots n+m\}: \text { Real } f^{*}\left(f_{i}\right) f_{i}^{*}(f) \geq 0\right\}
$$

and

$$
\Omega_{2}(f)=\left\{i \in\{n+1, n+2, \cdots n+m\}: \operatorname{Real} f^{*}\left(f_{i}\right) f_{i}^{*}(f)<0\right\},
$$

where $n \geq n_{0}=\max _{i \in \Omega^{\prime}} i, m \geq 1$ and $f^{*} \in S_{\mathcal{X}^{*}}$ is arbitrary. Then, by using (4.4), we have

$$
\begin{aligned}
& \sum_{i=n+1}^{n+m}\left|\operatorname{Real} f^{*}\left(f_{i}\right) f_{i}^{*}(f)\right| \\
& \quad=\sum_{j=1}^{2} \sum_{i \in \Omega_{j}\left(f^{*}\right)}\left|\operatorname{Real} f^{*}\left(f_{i}\right) f_{i}^{*}(f)\right| \\
& \quad=\sum_{j=1}^{2}\left|\operatorname{Real} f^{*}\left(\sum_{i \in \Omega_{j}(f)} f_{i}^{*}(f) f_{i}\right)\right| \\
& \quad \leq \sum_{j=1}^{2}\left\|f^{*}\right\|\left\|\sum_{i \in \Omega_{j}(f)} f_{i}^{*}(f) f_{i}\right\| \\
& \quad \leq \sum_{j=1}^{2}\left(\left\|f-\sum_{i \in \Omega_{j}(f) \cup \Omega} f_{i}^{*}(f) f_{i}\right\|+\left\|f-\sum_{i \in \Omega_{j}(f) \cup \Omega} f_{i}^{*}(f) f_{i}\right\|\right) \\
& \left.\quad<\frac{\epsilon}{2} \text { for all } f \in \mathcal{X}_{\text {and for all } f^{*} \in S_{\mathcal{X}^{*} .}} \|\right)
\end{aligned}
$$

Similarly we can show that

$$
\sum_{i=n+1}^{n+m}\left|\operatorname{Im} f^{*}\left(f_{i}\right) f_{i}^{*}(f)\right|<\frac{\epsilon}{2} \text { for all } f \in \mathcal{X} \text { and for all } f^{*} \in S_{\mathcal{X}^{*}} .
$$

By using (4.5) and (4.6), the theorem is proved. 


\section{Absolute type Reconstruction Property in Banach Spaces}

Definition 5.1. Suppose that $\left\{f_{k}^{*}\right\}$ has the reconstruction property for $\mathcal{X}$ with respect to $\left\{f_{k}\right\}$. We say that $\left(\left\{f_{k}\right\},\left\{f_{k}^{*}\right\}\right)$ is absolute, if the series

$$
\sum_{k=1}^{\infty} f_{k}^{*}(f) f_{k}
$$

is absolutely convergent for all $f \in \mathcal{X}$. That is, if $\sum_{k=1}^{\infty}\left\|f_{k}^{*}(f) f_{k}\right\|$ converges in $\mathbb{R}$ (the set of all real numbers) for all $f \in \mathcal{X}$.

Example 5.2. Let $\mathcal{X}=L^{1}\left(\Omega_{o}, \mu\right)$, where $\Omega_{o}=\mathbb{N}$ and $\mu$ is the counting measure and let $\chi_{k}=\{0,0, \cdots, \underbrace{1}_{k t h}, 0,0,0, \cdots\}(k \in \mathbb{N})$.

Define $\left\{f_{k}^{*}\right\} \subset \mathcal{X}^{*}$ by

$$
f_{1}^{*}(f)=0 \text { and } f_{k}^{*}(f)=\xi_{k-1}, k>1\left(f=\left\{\xi_{j}\right\} \in \mathcal{X}\right) .
$$

Choose $f_{1}=\chi_{1}$ and $f_{k}=\chi_{k-1}, k>1$. Then, $\left\{f_{k}^{*}\right\}$ has the reconstruction property for $\mathcal{X}$ with respect to $\left(\left\{f_{k}\right\}\right.$ which is absolute.

Example 5.3. Let $\mathcal{X}=\left(c_{0},\|.\|_{\infty}\right)$ and let $\left\{\chi_{k}\right\} \subset \mathcal{X}$ be the sequence of canonical unit vectors given in Example 5.2. Define $\left\{f_{k}^{*}\right\} \subset \mathcal{X}^{*}$ by

$$
f_{1}^{*}(f)=\xi_{1} \text { and for } k>1, f_{k}^{*}(f)=\xi_{k-1}, f=\left\{\xi_{j}\right\} \in \mathcal{X} .
$$

Choose $f_{1}=0$ and $f_{k}=\chi_{k-1}, k>1$. Then, $\left\{f_{k}^{*}\right\}$ has the reconstruction property for $\mathcal{X}$ with respect to $\left\{f_{k}\right\}$ which is not absolute.

Definition 5.4. Suppose that $\left(\left\{f_{k}\right\},\left\{f_{k}^{*}\right\}\right)$ has the reconstruction property for a Banach space $\mathcal{X}$. Then, $\left(\left\{f_{k}\right\},\left\{f_{k}^{*}\right\}\right)$ is said to be

(1) pre-positively confined, if there exist positive constants $\alpha$ and $\beta$ such that

$$
\alpha \leq\left\|f_{k}\right\| \leq \beta \text { for all } k \in \mathbb{N} .
$$

(2) post-positively confined, if there exist positive constants $a^{0}$ and $b^{0}$ such that

$$
a^{0} \leq\left\|f_{k}^{*}\right\| \leq b^{0} \text { for all } k \in \mathbb{N} .
$$

(3) positively confined, if it is both pre and post-positively confined.

The following theorem provides necessary and sufficient conditions for the prepositive confined reconstruction property in a Banach space to be absolute.

Theorem 5.5. Assume that $\left\{f_{k}^{*}\right\}$ has the reconstruction property for $\mathcal{X}$ with respect to $\left\{f_{k}\right\}$ which is pre-positively confined. Then, $\left(\left\{f_{k}\right\},\left\{f_{k}^{*}\right\}\right)$ is absolute if and only if the series $\sum_{k=1}^{\infty}\left|f_{k}^{*}(f)\right|$ converges for all $f \in \mathcal{X}$.

Proof. Since $\left(\left\{f_{k}\right\},\left\{f_{k}^{*}\right\}\right)$ is pre-positively confined, we can find positive constant $\alpha_{0}$ and $\beta_{0}$ such that $0<\alpha_{0} \leq\left\|f_{k}\right\| \leq \beta_{0}$ for all $k \in \mathbb{N}$.

Suppose first that $\left(\left\{f_{k}\right\},\left\{f_{k}^{*}\right\}\right)$ is absolute. Then, for all $f \in \mathcal{X}$, we have

$$
\sum_{k=1}^{\infty}\left|f_{k}^{*}(f)\right|=\sum_{k=1}^{\infty}\left\|\frac{f_{k}^{*}(f) f_{k}}{\left\|f_{k}\right\|}\right\|
$$




$$
\begin{aligned}
& \leq \alpha \sum_{k=1}^{\infty}\left\|f_{k}^{*}(f) f_{k}\right\|, \quad\left(\alpha=\frac{1}{\alpha_{0}}\right) \\
& <\infty
\end{aligned}
$$

For the reverse part, assume that the series $\sum_{k=1}^{\infty}\left|f_{k}^{*}(f)\right|$ converges for all $f \in \mathcal{X}$. We compute

$$
\begin{aligned}
\sum_{k=n}^{m}\left\|f_{k}^{*}(f) f_{k}\right\| & =\sum_{k=n}^{m}\left|f_{k}^{*}(f)\right|\left\|f_{k}\right\| \\
& \leq \beta_{0} \sum_{k=n}^{m}\left|f_{k}^{*}(f)\right| \rightarrow 0, \text { as } m, n \rightarrow \infty .
\end{aligned}
$$

Therefore, $\sum_{k=1}^{\infty}\left\|f_{k}^{*}(f) f_{k}\right\|$ converges in $\mathbb{R}$ for all $f \in \mathcal{X}$. Hence $\left(\left\{f_{k}\right\},\left\{f_{k}^{*}\right\}\right)$ is absolute.

Remark 5.6. The condition that the reconstruction system $\left(\left\{f_{k}\right\},\left\{f_{k}^{*}\right\}\right)$ is prepositively confined in Theorem 5.5 can not be relaxed.

Remark 5.7. Assume that $\left\{f_{k}^{*}\right\}$ has the reconstruction property for $\mathcal{X}$ with respect to $\left\{f_{k}\right\}$ which is positively confined. Then, dual version of Theorem 5.5 can be proved, provided the dual space of the underlying space admits the reconstruction property.

The following theorem gives a necessary condition for the reconstruction system to be absolute. More precisely, a topological isomorphism can be set up between the underlying space and $\ell^{1}$, provided $\left\{f_{k}\right\}$ is $\omega$-linearly independent, where $\left(\left\{f_{k}\right\},\left\{f_{k}^{*}\right\}\right)$ has the absolute reconstruction property for a Banach space $\mathcal{X}$. Recall that a sequence $\left\{f_{k}\right\} \subset \mathcal{X}$ is said to be $\omega$-linearly independent if $\sum_{k=1}^{\infty}\left|c_{k}\right|>0$ is impossible, whenever $\sum_{k=1}^{\infty} c_{k} f_{k}=0$.

Theorem 5.8. Suppose that $\left\{f_{k}^{*}\right\}$ has the absolute reconstruction property for $\mathcal{X}$ with respect to $\left\{f_{k}\right\}$. If $\left\{f_{k}\right\}$ is $\omega$-linearly independent, then there is a topological isomorphism of $\ell^{1}$ onto $\mathcal{X}$.

Proof. Define $\Theta: \ell^{1} \rightarrow \mathcal{X}$ by

$$
\Theta\left(\left\{\xi_{k}\right\}\right)=\sum_{k=1}^{\infty} \frac{\xi_{k} f_{k}}{\left\|f_{k}\right\|},\left\{\xi_{k}\right\} \in \ell^{1} .
$$

Then, for all $\left\{\xi_{k}\right\} \in \ell^{1}$, we have

$$
\begin{aligned}
\left\|\Theta\left(\left\{\xi_{k}\right\}\right)\right\| & =\left\|\sum_{k=1}^{\infty} \frac{\xi_{k} f_{k}}{\left\|f_{k}\right\|}\right\| \\
& \leq \sum_{k=1}^{\infty}\left|\xi_{k}\right| \quad\left(=\left\|\left\{\xi_{k}\right\}\right\|_{\ell^{1}}\right) \\
& <\infty
\end{aligned}
$$

Therefore, $\Theta$ is a bounded linear operator. Furthermore, $\operatorname{Ker} \Theta=\{0\}$ (where $\operatorname{Ker} \Theta$ denote the kernel of $\Theta$ ) is follows from the fact that $\left\{f_{k}\right\}$ is $\omega$-linearly 
independent. To show the ontoness of $\Theta$, let $f \in \mathcal{X}$ be arbitrary. Then, $f=$ $\sum_{k=1}^{\infty} f_{k}^{*}(f) f_{k}$. Choose $\eta_{k}=f_{k}^{*}(f)\left\|f_{k}\right\|$ for all $k \in \mathbb{N}$. Then, since $\left(\left\{f_{k}\right\},\left\{f_{k}^{*}\right\}\right)$ is absolute, we have

$$
\sum_{k=1}^{\infty}\left|\eta_{k}\right|=\sum_{k=1}^{\infty}\left\|f_{k}^{*}(f) f_{k}\right\|<\infty .
$$

Therefore, $\left\{\eta_{k}\right\} \in \ell^{1}$ is such that

$$
\begin{aligned}
\Theta\left(\left\{\eta_{k}\right\}\right) & =\sum_{k=1}^{\infty} \frac{\eta_{k} f_{k}}{\left\|f_{k}\right\|} \\
& =\sum_{k=1}^{\infty} \frac{f_{k}^{*}(f)\left\|f_{k}\right\| f_{k}}{\left\|f_{k}\right\|} \\
& =\sum_{k=1}^{\infty} f_{k}^{*}(f) f_{k} \\
& =f .
\end{aligned}
$$

Thus, $\Theta$ is onto. Therefore, by using the Open Mapping Theorem, $\Theta^{-1}$ is also bounded. Hence $\Theta$ is a topological isomorphism of $\ell^{1}$ onto $\mathcal{X}$.

Remark 5.9. If there is a topological isomorphism from a Banach space $\mathcal{X}$ onto $\ell^{1}$, then $\mathcal{X}$ has the reconstruction property. Indeed, let $\Theta_{0}: \ell^{1} \rightarrow \mathcal{X}$ be topological isomorphism. Let $\left\{\chi_{k}\right\}$ be the canonical unit vector basis (Schauder) for $\ell^{1}$. Choose $f_{k}=\Theta_{0}\left(\chi_{k}\right)$ and $f_{k}^{*}(f)=\xi_{k}$ for all $k \in \mathbb{N}$, where $f \in \mathcal{X}$ and $\left\{\xi_{k}\right\}$ is the sequence of scalars which appear in the infinite linear expansion of $\Theta_{0}^{-1}(f)$. Then, $\left\{f_{k}^{*}\right\}$ has the reconstruction property for $\mathcal{X}$ with respect to $\left\{f_{k}\right\}$.

Remark 5.10. If $\left(\left\{f_{k}\right\},\left\{f_{k}^{*}\right\}\right)$ has the absolute reconstruction property for a Banach space $\mathcal{X}$, then it is unconditional.

The following theorem shows that if $\mathcal{X}$ and $\mathcal{Y}$ are Banach spaces with absolute type reconstruction property, then their product $\mathcal{X} \times \mathcal{Y}$ with suitable norm also has the absolute reconstruction property.

Theorem 5.11. Suppose that $\left(\left\{f_{k}\right\},\left\{f_{k}^{*}\right\}\right)$ and $\left(\left\{g_{k}\right\},\left\{g_{k}^{*}\right\}\right)$ has the absolute reconstruction property for Banach spaces $\mathcal{X}$ and $\mathcal{Y}$, respectively. Then, there exists a sequence $\left\{h_{k}^{*}\right\} \subset(\mathcal{X} \times \mathcal{Y})^{*}$ such that $\left\{h_{k}^{*}\right\}$ has the absolute reconstruction property for $\mathcal{X} \times \mathcal{Y}$ with respect to some $\left\{h_{k}\right\} \subset \mathcal{X} \times \mathcal{Y}$.

Proof. Let $h=(f, g) \in \mathcal{X} \times \mathcal{Y}$ be arbitrary, where $f \in \mathcal{X}, g \in \mathcal{Y}$. Define $\left\{h_{k}\right\} \subset \mathcal{X} \times \mathcal{Y}$ and $\left\{h_{k}^{*}\right\} \subset(\mathcal{X} \times \mathcal{Y})^{*}$ by

$$
\begin{aligned}
& \left\{\begin{array}{l}
h_{2 k}=\left(0, g_{k}\right) \\
h_{2 k-1}=\left(f_{k}, 0\right)
\end{array}, k \in \mathbb{N} ;\right. \\
& \left\{\begin{array}{l}
h_{2 k}^{*}(f, g)=g_{k}^{*}(g) \\
h_{2 k-1}^{*}(f, g)=f_{k}^{*}(f)
\end{array}, k \in \mathbb{N} .\right.
\end{aligned}
$$


Then

$$
\begin{aligned}
h & =(f, g) \\
& =\left(\sum_{k=1}^{\infty} f_{k}^{*}(f) f_{k}, \sum_{k=1}^{\infty} g_{k}^{*}(g) g_{k}\right) \\
& =\sum_{k=1}^{\infty} h_{k}^{*}(f, g) h_{k} \\
& =\sum_{k=1}^{\infty} h_{k}^{*}(h) h_{k} \text { for all } h \in \mathcal{X} \times \mathcal{Y} .
\end{aligned}
$$

Thus, $\left\{h_{k}^{*}\right\}$ has the reconstruction property for $\mathcal{X} \times \mathcal{Y}$ with respect to $\left\{h_{k}\right\}$.

Now $\left(\left\{f_{k}\right\},\left\{f_{k}^{*}\right\}\right)$ and $\left(\left\{g_{k}\right\},\left\{g_{k}^{*}\right\}\right)$ are the absolute reconstruction property for $\mathcal{X}$ and $\mathcal{Y}$, respectively. Therefore, the series $\sum_{k=1}^{\infty}\left\|f_{k}^{*}(f) f_{k}\right\|$ converges for each $f \in \mathcal{X}$ and the series $\sum_{k=1}^{\infty}\left\|g_{k}^{*}(g) g_{k}\right\|$ converges for each $g \in \mathcal{Y}$. Thus, by the nature of construction of the system $\left(\left\{h_{k}\right\},\left\{h_{k}^{*}\right\}\right)$, the series $\sum_{k=1}^{\infty}\left\|h_{k}^{*}(h) h_{k}\right\|$ converges for all $h \in \mathcal{X} \times \mathcal{Y}$. Hence $\left\{h_{k}^{*}\right\}$ has the absolute reconstruction property for $\mathcal{X} \times \mathcal{Y}$ with respect to $\left\{h_{k}\right\}$.

To conclude the section, we discuss a special type of perturbation of the reconstruction property in Banach spaces. Suppose $\left\{f_{k}^{*}\right\}$ has the reconstruction property for $\mathcal{X}$ with respect to $\left\{f_{k}\right\}$. Then, in general, $\left\{f_{k}^{*} \pm(\bullet)\right\}$ does not has the reconstruction property for the underlying space with respect to any system $\left\{g_{k}\right\}$. The following proposition provides a sufficient condition for a particular type of a perturbed system to be the reconstruction property for the underlying space with respect to a certain system.

Proposition 5.12. Assume that $\left\{f_{k}^{*}\right\}$ has the reconstruction property for $\mathcal{X}$ with respect to $\left\{f_{k}\right\}$. Let $\epsilon>0$ be arbitrary and let $\left\{g_{k}^{*}\right\} \subset \mathcal{X}^{*}$ be a sequence given by

$$
g_{k}^{*}=\frac{1}{\left|f_{k}^{*}\left(f_{0}\right)\right|+\epsilon} f_{k}^{*}-\frac{1}{\left|f_{k+1}^{*}\left(f_{0}\right)\right|+\epsilon} f_{k+1}^{*} \text { for all } k \in \mathbb{N},
$$

where $f_{0} \in \mathcal{X}$ is fixed. Then, $\left\{g_{k}^{*}\right\}$ has the reconstruction property for $\mathcal{X}$ with respect to

$$
\left\{\sum_{k=1}^{n}\left(f_{k}^{*}\left(f_{0}\right)+\epsilon\right) f_{k}\right\},
$$

provided $\frac{f_{n+1}^{*}(f)}{\left|f_{n+1}^{*}\left(f_{0}\right)\right|+\epsilon} g_{n} \rightarrow 0$ as $n \rightarrow \infty$ in the norm for all $f \in \mathcal{X}$.

Proof. Choose $g_{n}=\sum_{k=1}^{n}\left(f_{k}^{*}\left(f_{0}\right)+\epsilon\right) f_{k}$ for all $n \in \mathbb{N}$. Then, for all $f \in \mathcal{X}$, we have

$$
\begin{aligned}
\sum_{k=1}^{n} g_{k}^{*}(f) g_{k} & =\sum_{k=1}^{n} f_{k}^{*}(f) f_{k}-\frac{f_{n+1}^{*}(f)}{\left|f_{n+1}^{*}\left(f_{0}\right)\right|+\epsilon} g_{n} \\
& \longrightarrow f \text { in the norm as } n \rightarrow \infty
\end{aligned}
$$


Therefore, $f=\sum_{k=1}^{\infty} g_{k}^{*}(f) g_{k}$ for all $f \in \mathcal{X}$. Hence $\left\{g_{k}^{*}\right\}$ has the reconstruction property for $\mathcal{X}$ with respect to $\left\{g_{k}\right\}$.

\section{Frames Related to the Reconstruction Property in Banach SPACES}

The reconstruction property in Banach spaces is a source of other types of associated redundant reconstruction systems and frames. For example, if $\left(\left\{f_{k}\right\},\left\{f_{k}^{*}\right\}\right)$ has the reconstruction property for $\mathcal{X}$, then we can find a reconstruction operator $\mathcal{S}$ such that $\mathcal{F} \equiv\left(\left\{f_{k}^{*}\right\}, \mathcal{S}\right)$ is a Banach frame for $\mathcal{X}$. The Banach frame $\mathcal{F}$ is known as a Banach frame associated with $\left(\left\{f_{k}\right\},\left\{f_{k}^{*}\right\}\right)$. It would be interesting to know whether the reconstruction property (may be with additional hypotheses) provides frames or reconstruction systems for a wider class of Banach spaces. At present, we focus on the reconstruction property for a Banach space $\mathcal{X}$ which is pre-shrinking. It is observed that this special type of the reconstruction property is responsible for the existence of Banach frames for a class of compact operators on the underlying space. Recall that $\mathcal{K}(\mathcal{X})$ denote the family of all compact linear operators on $\mathcal{X}$. We give a result which provides a construction of Banach frames for the conjugate of $\mathcal{K}(\mathcal{X})$ from the pre-shrinking reconstruction property for the Banach space $\mathcal{X}$.

Theorem 6.1. Assume that $\mathcal{X}$ has the pre-shrinking reconstruction property. Then, there exists a reconstruction operator $\Theta$ such that $\left(\left\{\pi\left(\Lambda_{k}\right)\right\}, \Theta\right)$ (where $\left.\left\{\Lambda_{k}\right\} \subset \mathcal{K}(\mathcal{X})\right)$ is a Banach frame for $\mathcal{K}(\mathcal{X})^{*}$.

Proof. Suppose that $\left\{f_{k}^{*}\right\}$ has the pre-shrinking reconstruction property for $\mathcal{X}$ with respect to $\left\{f_{k}\right\}$. Let $h \in \mathcal{X}$ be a non-zero vector and let $f^{*} \in \mathcal{X}^{*}$. Define a one-dimensional operator $T: \mathcal{X} \rightarrow \mathcal{X}$ by

$$
T(f)=f^{*}(f) h, f \in \mathcal{X} .
$$

Since the reconstruction property $\left(\left\{f_{k}\right\},\left\{f_{k}^{*}\right\}\right)$ is pre-shrinking, for each $\epsilon>0$, we can find index $n$ and $i$ such that

$$
\left\|h-f_{n}\right\|<\frac{\epsilon}{\left\|f^{*}\right\|+\frac{\epsilon}{\|h\|}}
$$

and

$$
\left\|f^{*}-f_{i}^{*}\right\|<\frac{\epsilon}{\|h\|}
$$

For $i, n \in \mathbb{N}$, define a system of operators $T_{i, n}: \mathcal{X} \rightarrow \mathcal{X}$ by

$$
T_{i, n}(f)=f_{i}^{*}(f) f_{n}, f \in \mathcal{X} .
$$

By using (6.1) and (6.2) for all $f \in \mathcal{X}$, we have

$$
\begin{aligned}
\left\|T_{i, n}(f)-T(f)\right\| & =\left\|f_{i}^{*}(f) f_{n}-f^{*}(f) h\right\| \\
& =\left\|f_{i}^{*}(f) f_{n}-f_{i}^{*}(f) h+f_{i}^{*}(f) h-f^{*}(f) h\right\| \\
& \leq\left(\left\|f_{i}^{*}\right\|\left\|f_{n}-h\right\|+\|h\|\left\|f_{i}^{*}-f^{*}\right\|\right)\|f\| \\
& \leq\left(\left[\left\|f^{*}\right\|+\frac{\epsilon}{\|h\|}\right] \frac{\epsilon}{\left.\left\|f^{*}\right\|+\frac{\epsilon}{\|h\|}+\|h\| \frac{\epsilon}{\|h\|}\right)\|f\| .}\right.
\end{aligned}
$$


Thus, for all $i, n \in \mathbb{N}$, we have

$$
\left\|T_{i, n}-T\right\| \leq 2 \epsilon
$$

Therefore, the family of operators $\left\{T_{i, n}\right\}_{i, n}$ on $\mathcal{X}$ is dense in the set of all onedimensional operators on $\mathcal{X}$. Also, the span of one-dimensional operators is the family of all finite-dimensional operators on $\mathcal{X}$. But the family of all finitedimensional operators on $\mathcal{X}$ is dense in $\mathcal{K}(\mathcal{X})$. Therefore, we can find a sequence $\left\{\Lambda_{k}\right\} \subset \mathcal{K}(\mathcal{X})$ such that $\mathcal{Z}_{d}=\left\{\left\{\pi\left(\Lambda_{k}\right)\left(\Lambda^{*}\right)\right\}: \Lambda^{*} \in \mathcal{K}(\mathcal{X})^{*}\right\}$ is a Banach space with the norm given by

$$
\left\|\left\{\pi\left(\Lambda_{k}\right)\left(\Lambda^{*}\right)\right\}\right\|_{\mathcal{Z}_{d}}=\left\|\Lambda^{*}\right\|_{\mathcal{K}(\mathcal{X})^{*}}, \Lambda^{*} \in \mathcal{K}(\mathcal{X})^{*} .
$$

Define $\Theta: \mathcal{Z}_{d} \rightarrow \mathcal{K}(\mathcal{X})^{*}$ by $\Theta\left(\left\{\pi\left(\Lambda_{k}\right)\left(\Lambda^{*}\right)\right\}\right)=\Lambda^{*}, \Lambda^{*} \in \mathcal{K}(\mathcal{X})^{*}$. Then, $\Theta$ is a bounded linear operator such that $\left(\left\{\Lambda_{k}\right\}, \Theta\right)$ is a Banach frame for $\mathcal{K}(\mathcal{X})^{*}$.

Remark 6.2. If $\left\{f_{k}^{*}\right\}$ has the pre-shrinking reconstruction property for a Banach space $\mathcal{X}$ with respect to $\left\{f_{k}\right\}$, then exists a system $\left\{\Lambda_{k}^{*}\right\} \subset \mathcal{K}(\mathcal{X})^{*}$ and reconstruction operator $\mathcal{S}: \mathcal{Z}_{d_{0}} \rightarrow \mathcal{K}(\mathcal{X})$ such that $\left(\left\{\Lambda_{k}^{*}\right\}, \mathcal{S}\right)$ is a Banach frame for $\mathcal{K}(\mathcal{X})$ with respect to $\mathcal{Z}_{d_{0}}$.

To conclude the paper we show that a frame type inequality is satisfied by the reconstruction property in Banach spaces.

Theorem 6.3. Suppose that $\left\{f_{k}^{*}\right\}$ has the reconstruction property for a Banach space $\mathcal{X}$ with respect to $\left\{f_{k}\right\}$. Then, there exists finite positive constants $A_{0}, B_{0}$ such that

$$
A_{0}\left\|f^{*}\right\| \leq \sup _{1 \leq n<\infty}\left\|\sum_{k=1}^{n} f^{*}\left(f_{k}\right) f_{k}^{*}\right\| \leq B_{0}\left\|f^{*}\right\| \text { for all } f^{*} \in \mathcal{X}^{*} .
$$

Proof. For arbitrary $f^{*} \in \mathcal{X}^{*}$, we compute

$$
\begin{aligned}
\sup _{1 \leq n<\infty}\left\|\sum_{k=1}^{n} f^{*}\left(f_{k}\right) f_{k}^{*}\right\| & =\sup _{1 \leq n<\infty}\left\|\mathcal{P}_{n}^{*}\left(f^{*}\right)\right\| \\
& \leq \sup _{1 \leq n<\infty}\left\|\mathcal{P}_{n}^{*}\right\|\left\|f^{*}\right\| \\
& =\sup _{1 \leq n<\infty}\left\|\mathcal{P}_{n}\right\|\left\|f^{*}\right\| \\
& =B_{0}\left\|f^{*}\right\|,
\end{aligned}
$$

where $\left\{\mathcal{P}_{n}\right\}$ is a sequence of finite rank operators on $\mathcal{X}$ defined by $\mathcal{P}_{n}(f)=$ $\sum_{k=1}^{n} f_{k}^{*}(f) f_{k}, f \in \mathcal{X}$ and $B_{0}=\sup _{1 \leq n<\infty}\left\|\mathcal{P}_{n}\right\|<\infty$.

Let $\epsilon>0$ and $f^{*} \in \mathcal{X}^{*}$ be arbitrary nonzero functional. Then, we can find $f \in \mathcal{X}$ such that $\|f\|=1$ and $\left\|f^{*}\right\|-\epsilon \leq\left|f^{*}(f)\right|$ (note that $f \leftrightarrow f_{\epsilon, f^{*}}$ ).

For this $f$, we compute

$$
\begin{aligned}
\left\|f^{*}\right\| & \leq\left|f^{*}(f)\right|+\epsilon \\
& =\left|f^{*}\left(\sum_{k=1}^{\infty} f_{k}^{*}(f) f_{k}\right)\right|+\epsilon
\end{aligned}
$$




$$
\begin{aligned}
& =\lim _{n \rightarrow \infty}\left|\left(\sum_{k=1}^{n} f^{*}\left(f_{k}\right) f_{k}^{*}\right)(f)\right|+\epsilon \\
& \leq \sup _{1 \leq n<\infty}\left|\left(\sum_{k=1}^{n} f^{*}\left(f_{k}\right) f_{k}^{*}\right)(f)\right|+\epsilon \\
& \leq \sup _{1 \leq n<\infty}\left\|\sum_{k=1}^{n} f^{*}\left(f_{k}\right) f_{k}^{*}\right\|\|f\|+\epsilon \\
& =\sup _{1 \leq n<\infty}\left\|\sum_{k=1}^{n} f^{*}\left(f_{k}\right) f_{k}^{*}\right\|+\epsilon .
\end{aligned}
$$

Note that (6.4) is also true for $f^{*}=0$

Since $\epsilon>0$ and $f^{*} \in \mathcal{X}^{*}$ were arbitrary. Therefore, by using (6.4), we have

$$
\left\|f^{*}\right\| \leq \sup _{1 \leq n<\infty}\left\|\sum_{k=1}^{n} f^{*}\left(f_{k}\right) f_{k}^{*}\right\| .
$$

By using (6.3) and (6.5), with $A_{0}=1$ and $B_{0}=\sup _{1 \leq n<\infty}\left\|\mathcal{P}_{n}\right\|$, we have

$$
A_{0}\left\|f^{*}\right\| \leq \sup _{1 \leq n<\infty}\left\|\sum_{k=1}^{n} f^{*}\left(f_{k}\right) f_{k}^{*}\right\| \leq B_{0}\left\|f^{*}\right\| \text { for all } f^{*} \in \mathcal{X}^{*} .
$$

The theorem is proved.

Acknowledgement. The second author is partly supported by R \& D Doctoral Research Programme, University of Delhi, Delhi. Grant No. DRCH/R\&D/2013$14 / 4155$.

\section{REFERENCES}

1. P.G. Casazza and G. Kutyniok, Finite Frames: Theory and Applications, Birkhäuser, 2012.

2. P.G. Casazza, D. Han and D.R. Larson, Frames for Banach spaces, Contemp. Math. 247 (1999), 149-182.

3. P.G. Casazza, Local thoery of frames and Schauder bases for Hilbert spaces, Illinois J. Math. 43 (1999), 291-306.

4. P.G. Casazza, Finite dimensional decompositions in Banach spaces, Cont. Math. 52 (1986), $1-31$.

5. P.G. Casazza, S.J. Dilworth and E. Odell, Th. Schlumprecht and A. Zsák, Coefficient quantization for frames in Banach spaces, J. Math. Anal. Appl. 348 (2008), 66-86.

6. P.G. Casazza and N.J. Kalton, Uniqueness of unconditional bases in Banach spaces, Israel J. Math. 103 (1998), 141-171.

7. P.G. Casazza and N.J. Kalton, Unconditional bases and unconditional finite dimensional decompositions in Banach spaces, Israel J. Math. 95 (1996), 349-373.

8. P.G. Casazza, Approximation Properties, in Handbook on the Geometry of Banach spaces Vol. I, W. B. Johnson and J. Lindenstrauss editors, 2001, 271-316.

9. P.G. Casazza and B. Lin, On Conditional Bases in Banach Spaces, Revue Roumaine Math. Pures et App. 19 (1974), 745-754.

10. P.G. Casazza and N.J. Kalton, Notes on Approximation properties in Separable Banach spaces, 49-65, Lecture notes 158, London Math. Soc., 1991. 
11. P.G. Casazza and O. Christensen, The reconstruction property in Banach spaces and a perturbation theorem, Canad. Math. Bull. 51 (2008), 348-358.

12. R.R. Coifman and G. Weiss, Expansions of Hardy spaces and their use in analysis, Bull. Amer. Math. Soc. 83 (1977), 569-645.

13. R.J. Duffin and A.C. Schaeffer, A class of nonharmonic Fourier series, Trans. Amer. Math. Soc. 72 (1952), 341-366.

14. I. Daubechies, A. Grossmann and Y. Meyer, Painless non-orthogonal expansions, J. Math. Phys. 27 (1986), 1271-1283.

15. N. Dunford and J.T. Schwartz, Linear Operators: Part I, Interscience Publishers Inc., New York, 1958.

16. M. Fabian, P. Habala, P. Hájek, V.M. Santalucía, J. Pelant and V. Zizler, Functional Analysis and Infinite-Dimensional Geometry, Springer-Verlag, New York, 2001.

17. H. Feichtinger and K. Gröchenig, Banach spaces related to integrable group representations and their atomic decompositions, I, J. Funct. Anal. 86 (1989), no. 2, 307-340.

18. H. Feichtinger and K. Gröchenig, Banach spaces related to integrable group representations and their atomic decompositions, II, Monatsh. Math. 108 (1989), no. 2-3, 129-148.

19. D. Gabor, Theory of communicatons, J. Inst. Elec. Engg. 93 (1946), no. 3, 429-457.

20. K. Gröchenig, Describing functions: Atomic decompositions versus frames, Monatsh. Math. 112 (1991), 1-41.

21. D. Han and D.R. Larson, Frames, bases and group representations, Mem. Amer. Math. Soc. 147 (2000), no. 697.

22. C. Heil and D. Walnut, Continuous and discrete wavelet transforms, SIAM Rev. 31 (1989), 628-666.

23. W.B. Johnson and J. Lindenstrauss, Handbook of the Geometry of Banach Spaces: Vol. I, Elsevier, Amsterdam, 2001.

24. W.B. Johnson and J. Lindenstrauss, Handbook of the Geometry of Banach Spaces: Vol. II, Elsevier, Amsterdam, 2003.

25. W.B. Johnson, J. Lindenstrauss and G. Schechtman, On the relation between several notions of unconditional structure, Israel J. Math. 37 (1980), no. 1-2, 120-129.

26. S.K. Kaushik, L. K. Vashisht and G. Khattar, Reconstruction property and frames in Banach spaces, Palest. J. Math. 3 (2014), no. 1, 11-26.

27. S. Karlin, Bases in Banach spaces, Duke Math. J. 15 (1948), 971-985.

28. G. Khattar and L. K. Vashisht, The reconstruction property in Banach spaces generated by matrices, Adv. Pure Appl. Math. 5 (2014), no. 3, 151-160.

29. I. Singer, Bases in Banach Spaces, Vol. I, Springer, New York, 1981.

30. L.K. Vashisht and G. Khattar, On I-reconstruction property, Adv. Pure Math. 3 (2013), no. 3, 324-330.

Department of Mathematics, University of Delhi, Delhi-110007, India.

E-mail address: geetika1684@gmail.com

E-mail address: lalitkvashisht@gmail.com 